\title{
Benzodiazepines for Psychosis-Induced Aggression or Agitation
}

\author{
Hadar Zaman*,1, Stephanie Sampson², Alison Beck ${ }^{3}$, Tarang Sharma ${ }^{4}$, Fiona Clay ${ }^{5}$, Styliani Spyridi ${ }^{6,7}$, Sai Zhao ${ }^{8}$, and \\ Donna Gillies ${ }^{9}$
}

${ }^{1}$ Bradford School of Pharmacy and Medical Sciences, University of Bradford, Bradford, UK; ${ }^{2}$ Division of Psychiatry, Cochrane Schizophrenia Group, Nottingham, UK; ${ }^{3}$ Department of Psychology and Psychotherapy, South London and Maudsley NHS Foundation Trust, Trust HQ, London, UK; ${ }^{4}$ Public Health and Epidemiology, Nordic Cochrane Centre, Copenhagen, Denmark; ${ }^{5}$ Department of Forensic Medicine, Monash University, Melbourne, Australia; ${ }^{6}$ Cambridgeshire and Peterborough NHS Foundation Trust, Cambridge, UK; ${ }^{7}$ Department of Rehabilitation Sciences, Faculty of Health Sciences, Cyprus University of Technology, Lemesos, Cyprus; ${ }^{8}$ The Ingenuity Centre, The University of Nottingham, Systematic Review Solutions Ltd, Nottingham, UK; ${ }^{9}$ Mental Health Education Centre, Western Sydney Local Health District - Mental Health, Sydney, Australia

*To whom correspondence should be addressed; Bradford School of Pharmacy and Medical Sciences, University of Bradford, Great Horton Road, Bradford BD7 1DP, UK; tel: 441274 234741, e-mail: hzaman4@bradford.ac.uk

Key words: tranquilisation/adverse-effects/benzodiazepine

\section{Background}

Acute psychotic illness, especially when associated with agitated or violent behavior, can require urgent pharmacological tranquillization or sedation. In many countries, benzodiazepines (either alone or in combination with antipsychotics) are often used in this situation (1).

\section{Objectives}

To examine whether benzodiazepines, alone or in combination with other pharmacological agents, are an effective treatment for psychosis-induced aggression or agitation when compared with placebo, other pharmacological agents (alone or in combination) or non-pharmacological approaches.

\section{Search Methods}

We searched the Cochrane Schizophrenia Group's register (January 2012, August 20, 2015 and August 3, 2016), inspected reference lists of included and excluded studies, and contacted authors of relevant studies.

\section{Selection Criteria}

We included all randomized controlled trials (RCTs) comparing benzodiazepines alone or in combination (with antipsychotics), vs placebo or antipsychotics alone or in combination (with other antipsychotics, benzodiazepines or antihistamines) for people who were aggressive or agitated due to psychosis.

\section{Data Collection and Analysis}

We reliably selected studies, quality assessed them, and extracted data. For binary outcomes, we calculated standard estimates of risk ratio (RR) and their 95\% CI using a fixed-effect model. For continuous outcomes, we calculated the mean difference (MD) between groups. If there was heterogeneity, this was explored using a random-effects model. We assessed the risk of bias and created a "Summary of findings" table using GRADE (table 1).

\section{Main Results}

Twenty trials including 695 participants are included in this review. The quality of evidence for the main outcomes was low or very low due to the very small sample sizes of included studies and serious risk of bias (randomization, allocation concealment and blinding were not well conducted in the included trials, and 6 out of the 20 trials were supported by pharmaceutical institutes). There was no clear effect for most outcomes.

\section{Benzodiazepines Alone vs Placebo}

One trial compared benzodiazepines alone (IM lorazepam) with placebo. There was no difference in the number sedated at 24 hours (very low quality of evidence). However, more people receiving placebo showed no improvement in global state in the medium term ( 1 to 48 h) ( $n=102,1$ RCT, RR $0.62,95 \%$ CI 0.40 to 0.97 , very low quality of evidence). 


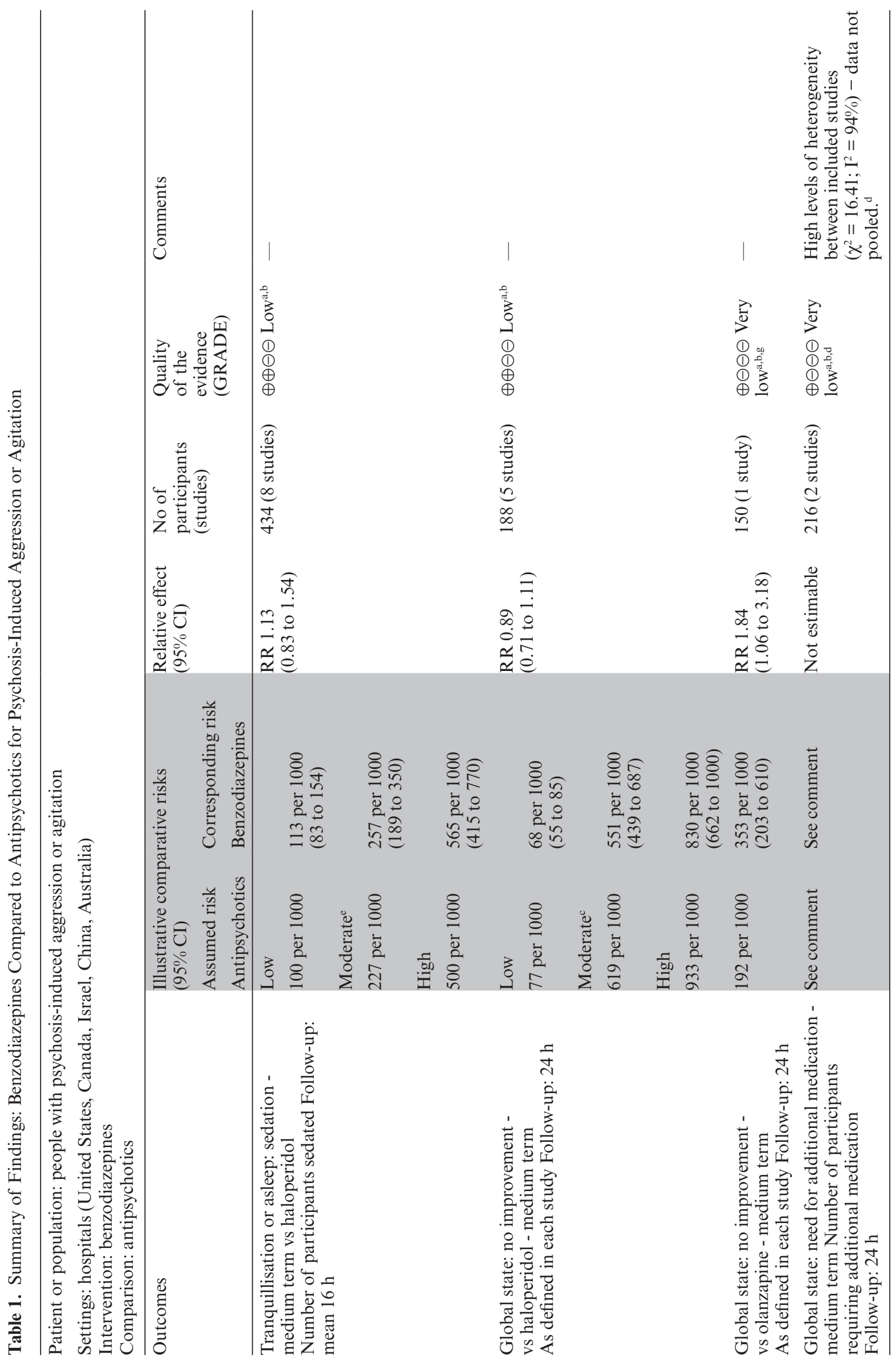

Page 2 of 4 


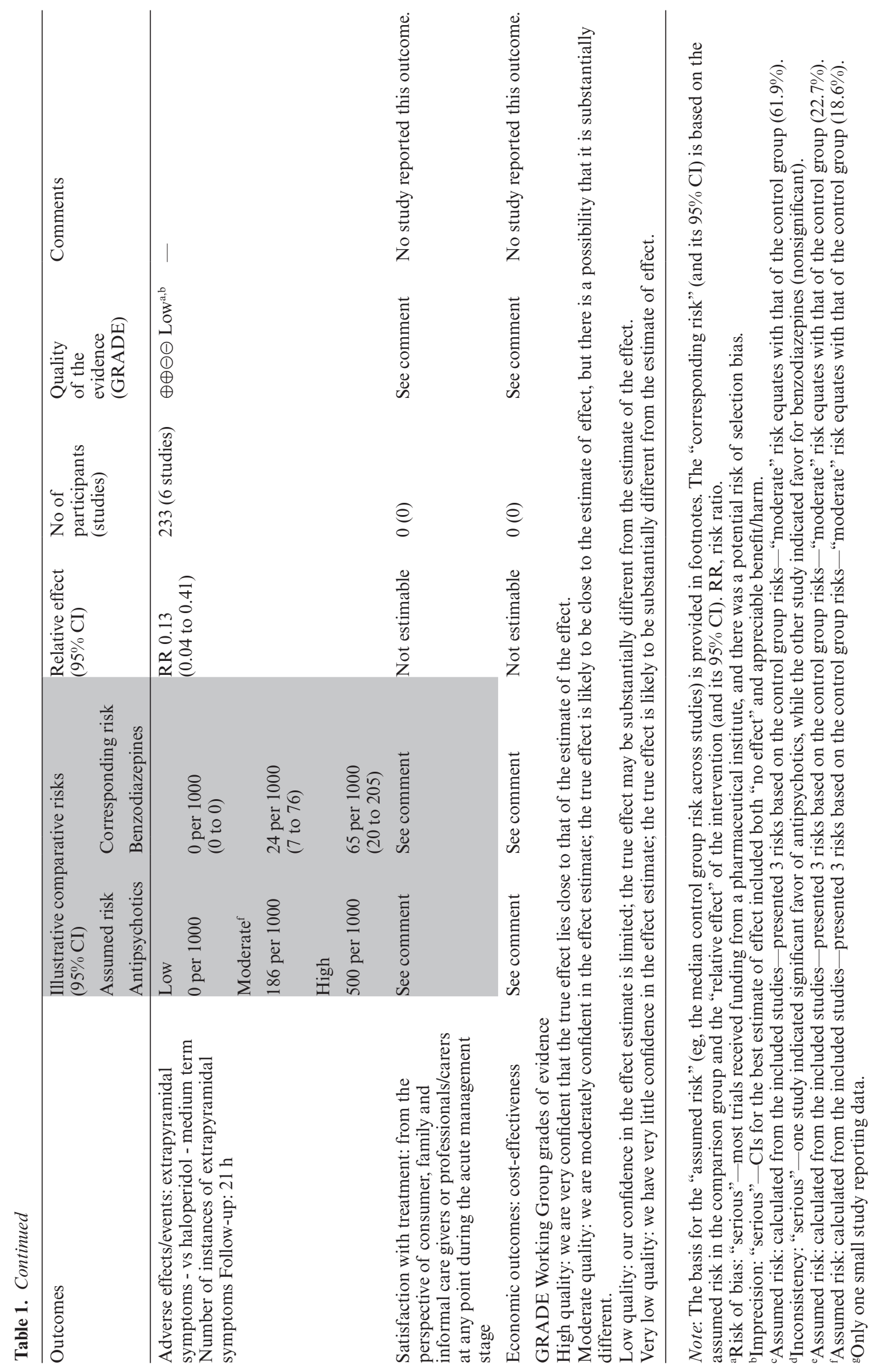

Page 3 of 4 


\section{Benzodiazepines Alone vs Antipsychotics}

Eleven trials compared benzodiazepines with antipsychotics. Compared with haloperidol there was no difference for sedation at 16 hours $(n=434,8$ RCTs, RR 1.13, $95 \%$ CI 0.83 to 1.54 , low quality of evidence) or improvement (global state) in the medium term $(n=188,5$ RCTs, RR $0.89,95 \%$ CI 0.71 to 1.11 , low quality of evidence).

In one small trial, fewer participants improved (global state) in the medium term when receiving lorazepam compared with olanzapine $(n=150,1$ RCT, RR 1.84, $95 \%$ CI 1.06 to 3.18 , very low quality of evidence).

People receiving benzodiazepines were less likely to experience extrapyramidal effects (EPS) in the medium term compared to people receiving haloperidol $(n=233$, 6 RCTs, RR $0.13,95 \%$ CI 0.04 to 0.41 , low quality of evidence).

\section{Benzodiazepines Alone vs Combined Antipsychotics/ Antihistamines}

When benzodiazepines (lorazepam or midazolam) were compared with combined antipsychotics/antihistamines (haloperidol plus promethazine), there was a higher risk of no improvement (global state) for benzodiazepines alone in the medium term $(n=200,1$ RCT, RR $2.17,95 \%$ CI 1.16 to 4.05 , low quality of evidence). However, for sedation in the medium term, the results were unclear: compared with combined antipsychotics/antihistamines, lorazepam led to a lower risk of sedation $(n=200,1$ RCT, RR $0.91,95 \%$ CI 0.84 to 0.98 , low quality of evidence); while, midazolam led to a higher risk of sedation $(n=200,1$ RCT, RR $1.13,95 \%$ CI 1.04 to 1.23 , low quality of evidence).

\section{Other Combinations}

Benzodiazepines (lorazepam or clonazepam) plus antipsychotics (haloperidol or risperidone) vs benzodiazepines alone did not yield any clear differences for global state. When comparing combined benzodiazepines/antipsychotics (a haloperidol combination in all studies) with haloperidol alone, there was no difference in mediumterm improvement for global state $(n=185,4$ RCTs, RR $1.17,95 \%$ CI 0.93 to 1.46 , low quality of evidence), but sedation was more likely in the short-term for people who received the combination therapy $(n=172,3$ RCTs, RR $1.75,95 \%$ CI 1.14 to 2.67 , very low quality of evidence). Only one trial compared combined benzodiazepines/antipsychotics with antipsychotics; however, this study did not report our primary outcomes. One small trial compared combined benzodiazepines/antipsychotics (midazolam and haloperidol) with combined antihistamines/ antipsychotics (promethazine and haloperidol). The combined benzodiazepines/antipsychotics group had a higher risk of no improvement (global state) $(n=60,1$ RCT, RR $25.00,95 \%$ CI 1.55 to 403.99 , very low quality of evidence) and higher levels of sedation in the medium term $(n=60,1$ RCT, RR $12.00,95 \%$ CI 1.66 to 86.59 , very low quality of evidence).

\section{Authors' Conclusions}

The small amount of evidence is of poor quality. Most trials were too small to highlight differences. A direct comparison of single agent benzodiazepines vs antipsychotics demonstrated a possible small advantage of antipsychotics. However, caution must be exercised as older antipsychotic agents also had a disadvantage in terms of side effects (EPS). Adding benzodiazepines to other drugs does not seem to confer clear advantage and has the potential for adding unnecessary adverse effects. It would appear that antihistamines would be a better choice of additive agent to antipsychotics than benzodiazepines; however, the quality of evidence was very low. Much more high-quality research is still needed in this area.

\section{Reference}

1. Zaman H, Sampson SJ, Beck ALS, et al. Benzodiazepines for psychosis-induced aggression or agitation. Cochrane Database Syst Rev. 2017;12:CD003079. doi:10.1002/14651858. CD003079.pub4 